\title{
Effectiveness of adjuvant trastuzumab in daily clinical practice
}

\author{
Erika Matos, Branko Zakotnik, Cvetka Grasic Kuhar \\ Institute of Oncology Ljubljana, Department of Medical Oncology, Ljubljana, Slovenia
}

Radiol Oncol 2014; 48(4): 403-407.

Received 30 August 2013

Accepted 14 October 2013

Correspondence to: Erika Matos, M.D., M.Sc., Institute of Oncology Ljubljana, Department of Medical Oncology, Zaloška 2, SI-1000 Ljubljana, Slovenia. E-mail. ematos@onko-i.si

Disclosure: No potential conflicts of interest were disclosed.

Background. Human epidermal growth factor receptor 2 (HER2) positive breast cancer is an entity with aggressive behaviour. One year of adjuvant trastuzumab significantly improves the disease free survival in the range of $40-50 \%$ and reduces the risk of dying from HER2 positive breast cancer by one third. Adjuvant treatment with trastuzumab became available in Slovenia in 2005 and the aim of this study is to explore, if the exceptional results reported in adjuvant clinical trials are achieved also in daily clinical practice.

Patients and methods. An analysis of tumour and patient characteristics, type of treatment and outcome (relapse free and overall survival) of 313 patients (median age 52 years) treated at the Institute of Oncology Ljubljana in years 2005-2009 was performed.

Results. Median follow-up was 4.4 years. Sixty-one patients relapsed and 24 died. Three and four years relapse free survival was $84.2 \%$ and $80.8 \%$ and the overall survival was $94.4 \%$ and $92.5 \%$, respectively. Independent prognostic factors for relapse were tumour grade (HR 2.10; $95 \% \mathrm{Cl} 1.07-4.14 ; \mathrm{p}=0.031$ ) and nodal stage (HR 1.35; 1.16-1.56; $p<0.0001$ ) and for the overall survival nodal stage only (HR 1.36; 1.05-1.78; $p=0.021)$.

Conclusions. The outcome in patients with adjuvant trastuzumab in daily clinical practice, treated by medical oncologists, is comparable to results obtained in international adjuvant studies.

Key words: breast cancer; trastuzumab, adjuvant; daily clinical practice

\section{Introduction}

The most common cancer in women in the developed world as well as in Slovenia is breast cancer (BC). ${ }^{1}$ With the introduction of the tumour gene signature the clinical observation that $\mathrm{BC}$ is a spectrum of different diseases in terms of prognosis and response to the treatment was confirmed. ${ }^{2}$ Using this tool as well as by classical clinico-pathological parameters four types of BCs can be distinguished and human epidermal growth factor receptor 2 (HER2) positive type is one of them, representing about $15 \%$ of newly diagnosed invasive BCs. ${ }^{2-4}$ It is a unique entity with an aggressive behaviour, characterized by overexpression of HER2 receptor and/or HER2 gene amplification. 3,5 At the beginning of this century, trastuzumab, a humanized monoclonal antibody, that targets HER2 receptor, was approved for the treatment of patients with metastatic HER2positive BC. ${ }^{6-8}$ Given the success of this antiHER2 drug in the metastatic setting, several large, randomized trials were initiated to evaluate its role in the early stage disease. The first results were presented in 2005 and were the basis for the approval of one year adjuvant treatment of patients with HER2 positive BC. ${ }^{9-11}$ A significant improvement in disease free survival (DFS) in the range of $40-50 \%$ was demonstrated and the risk from dying from BC was reduced by about one third. Reported 4-year DFS and the overall survival (OS) in the trastuzumab arms were 78.6-86\% and 89.3$94 \%$, respectively. ${ }^{12-15}$ This is the range of benefit seldom achieved in oncology. In the proceeding years new antiHER2 drugs confirmed their activity in metastatic setting; i.e. lapatinib, pertuzumab 
and trastuzumab-emtansine. ${ }^{16-18}$ Adjuvant studies with new antiHER2 drugs are in progress.

The adjuvant treatment with trastuzumab became available in Slovenia in 2005 and the aim of this report is to explore if these exceptional results reported in adjuvant clinical trials are achieved also in daily clinical practice.

\section{Patients and methods}

With the approval of the adjuvant trastuzumab treatment the Slovenian HER2 registry was set up. The criteria for the adjuvant treatment with trastuzumab regarding tumour and nodal stage and cardiac function were the same as in pivotal adjuvant trials: tumours larger then $2 \mathrm{~cm}$ if node negative disease, any tumour size if node positive disease, performance status zero or one, no serious concomitant cardiac diseases and treatment with adjuvant chemotherapy.9-11 Data were collected from patient's records. Patients were treated at the Institute of Oncology Ljubljana.

The study was approved by the institutional review board committed.

The main objective of this project was to evaluate the outcome of our real life patient population: relapse free survival (RFS) and OS. We compared our results with the results from randomized studies and other population-based studies.

\section{Statistical analysis}

RFS was defined as time elapsed from date of surgery to date of the first relapse (local or distant), date of the last follow-up or date of death without relapse. Patients who died without relapse were censored at time of death. OS was defined as time from surgery to date of death of any cause or date of the last follow-up for patients who were alive. The univariate statistical analysis was performed using Kaplan-Meier method and log-rank test. The multivariate analysis was performed with Cox proportional hazards model. SPSS software version 16 was used for the statistical analysis.

\section{Results}

In the 5-year period (2005-2009) 313 patients with HER2 positive BC were treated with adjuvant trastuzumab. The median age of the patients was 52 years (23-76). Median follow-up time was 4.4 years (minimum 0.2 years, maximum 6.9 years). The char-
TABLE 1. Tumour characteristics of 313 patients

\begin{tabular}{|c|c|c|c|}
\hline & & No. & \\
\hline \multirow{3}{*}{ Histology } & IDC & 297 & $95 \%$ \\
\hline & ILC & 6 & $2 \%$ \\
\hline & Other & 10 & $3 \%$ \\
\hline \multirow{4}{*}{$\begin{array}{l}\text { Tumor } \\
\text { grade }\end{array}$} & I & 3 & $1 \%$ \\
\hline & $\|$ & 84 & $27 \%$ \\
\hline & III & 220 & $70 \%$ \\
\hline & Unknown & 6 & $2 \%$ \\
\hline \multirow{4}{*}{$\begin{array}{l}\text { Mitotic } \\
\text { index }\end{array}$} & 1 & 44 & $14 \%$ \\
\hline & 2 & 94 & $30 \%$ \\
\hline & 3 & 131 & $42 \%$ \\
\hline & Unknown & 44 & $14 \%$ \\
\hline \multirow{3}{*}{$\begin{array}{l}\text { Vascular } \\
\text { invasion }\end{array}$} & Present & 15 & $5 \%$ \\
\hline & Absent & 186 & $60 \%$ \\
\hline & Unknown & 112 & $35 \%$ \\
\hline \multirow{6}{*}{$\begin{array}{l}\text { Hormonal } \\
\text { receptor } \\
\text { status }\end{array}$} & ER positive & 176 & $56 \%$ \\
\hline & ER negative & 137 & $44 \%$ \\
\hline & PR positive & 130 & $42 \%$ \\
\hline & PR negative & 180 & $58 \%$ \\
\hline & $\begin{array}{l}\text { ER and PR } \\
\text { negative }\end{array}$ & 126 & $40 \%$ \\
\hline & Unknown & 1 & $0 \%$ \\
\hline \multirow{6}{*}{$\begin{array}{l}\text { Tumour } \\
\text { stage }\end{array}$} & $\mathrm{Tl}$ & 88 & $28 \%$ \\
\hline & $\mathrm{T} 2$ & 152 & $48 \%$ \\
\hline & T3 & 34 & $11 \%$ \\
\hline & $\mathrm{T} 4$ & 8 & $3 \%$ \\
\hline & $\mathrm{T} 4 \mathrm{~d}$ & 25 & $8 \%$ \\
\hline & Unknown & 6 & $2 \%$ \\
\hline \multirow{5}{*}{$\begin{array}{l}\text { Nodal } \\
\text { stage }\end{array}$} & NO & 79 & $25 \%$ \\
\hline & $\mathrm{N} 1$ & 157 & $50 \%$ \\
\hline & N2 & 51 & $16 \%$ \\
\hline & N3 & 24 & $8 \%$ \\
\hline & Unknown & 2 & $1 \%$ \\
\hline
\end{tabular}

IDC = invasive ductal carcinoma; ILC = invasive lobular carcinoma

acteristics of the tumours are presented in Table 1. One hundred and twenty-seven (40\%) of patients received an anthracycline-based and 165 (53\%) anthracycline- and taxane-based chemotherapy. One hundred and seventy-six (56\%) of patients had estrogen receptor (ER) and $130(42 \%)$ of patients had progesterone receptor (PR) positive tumours. All patients with hormone dependent tumours (187 [60\%]) were also treated with adjuvant endocrine therapy. Two hundred and seven (66\%) patients 
TABLE 2. Relapse free survival (univariate analysis)

\begin{tabular}{lcc}
\hline & HR $(95 \% \mathrm{Cl})$ & P value \\
\hline Tumour stage & $1.25(1,11-1.40)$ & $<0.0001$ \\
Nodal stage & $1.42(1,23-1.64)$ & $<0.0001$ \\
ER status & $1.14(0,68-1.90)$ & 0.62 \\
PR status & $0.82(0,49-1.36)$ & 0.43 \\
Tumour grade & $1.91(0,98-3.72)$ & 0.059 \\
Mitotic index & $1.35(0,89-2.03)$ & 0.157 \\
Histological type* & $1.12(0,99-1.27)$ & 0.081 \\
Vascular invasion & $1.04(0,98-1.10)$ & 0.162 \\
Chemo - type** & $1.29(0.85-1.98)$ & 0.237 \\
\hline
\end{tabular}

* invasive ductal carcinoma, invasive lobular carcinoma, other types ** anthracycline-based, anthracycline- and taxane-based, other types of chemotherapy

TABLE 3. Relapse free survival (multivariate analysis). No. of events: $61 / 313$

\begin{tabular}{lcc}
\hline & HR $(95 \% \mathrm{Cl})$ & P VALUE \\
\hline Tumour grade & $2.10(1.07-4.14)$ & 0.031 \\
Nodal stage & $1.35(1.16-1.56)$ & $<0.0001$ \\
Tumour stage & $1.19(1.04-1.36)$ & 0.014 \\
\hline
\end{tabular}

TABLE 4. Overall survival (univariate analysis). No. of events: $24 / 313$

\begin{tabular}{lll}
\hline & HR $(95 \% \mathrm{CI})$ & P VALUE \\
\hline Tumour stage & $1.17(0,94-1,46)$ & 0.155 \\
Nodal stage & $1.36(1,05-1,78)$ & 0.021 \\
ER status & $1.22(0,54-2,80)$ & 0.633 \\
PR status & $0.87(0,39-1,96)$ & 0.733 \\
Tumour grade & $2.49(0,75-8,26)$ & 0.136 \\
Mitotic index & $1.63(0,82-3,24)$ & 0.162 \\
Histological type* & $1.12(0,49-1,35)$ & 0.211 \\
Vascular invasion & $1.01(0,92-1,11)$ & 0.821 \\
Chemo - type** & $0.84(0,40-1,74)$ & 0.632 \\
\hline
\end{tabular}

* invasive ductal carcinoma, invasive lobular carcinoma, other types ** anthracycline-based, anthracycline- and taxane-based, other types of chemotherapy

were concomitantly with trastuzumab irradiated to the chest wall, breast and supraclavicular region, according to the international guidelines. ${ }^{19}$

\section{RFS - relapse free survival}

Sixty-one patients (19.5\%) relapsed. Kaplan-Mayer curve for RFS is presented on Figure 1. RFS at 4

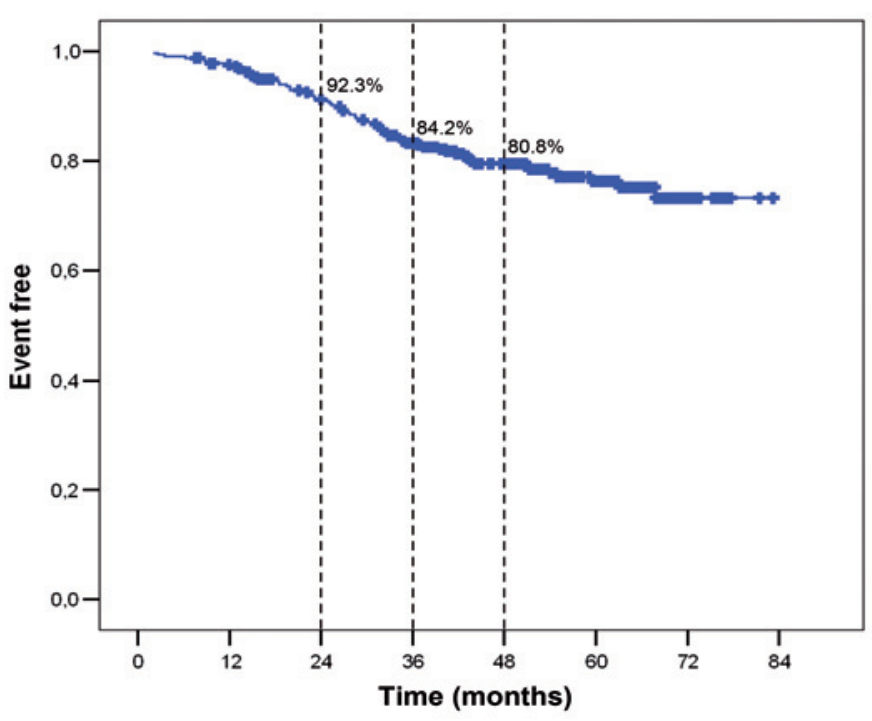

FIGURE 1. Relapse free survival (RFS).

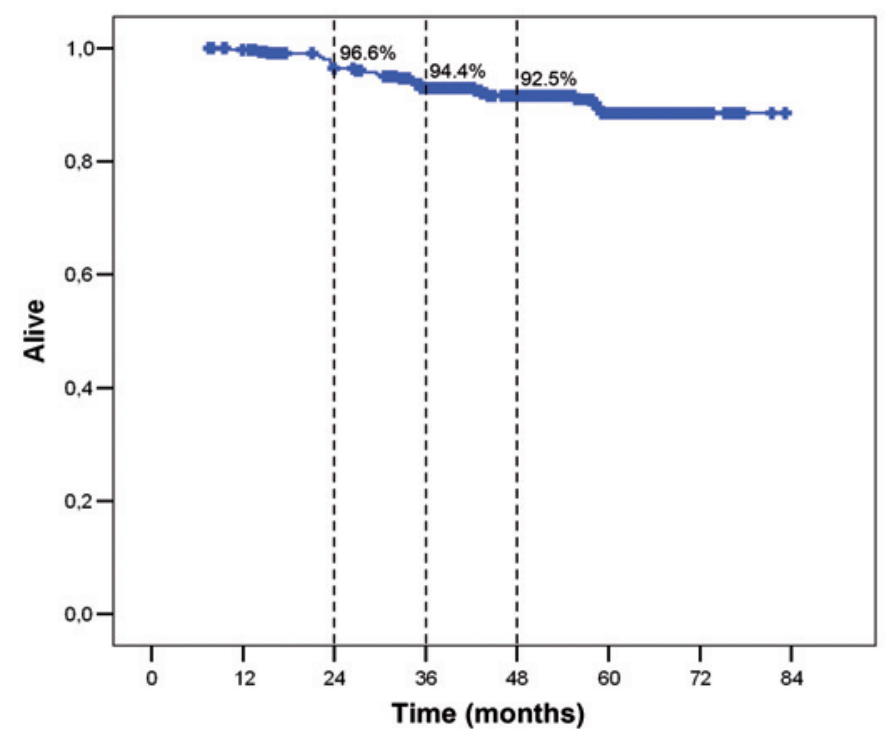

FIGURE 2. Overall survival (OS).

years was $80.8 \%$. Tumour stage and grade and nodal stage were found to have a significant impact on RFS in univariate analysis (Table 2). In the multivariate analysis only tumour grade (Hazard ratio [HR] 2.10) and nodal stage (HR 1.35) were found to have independent prognostic role (Table 3).

\section{OS - overall survival}

Twenty-four patients $(7.6 \%)$ died. Kaplan-Meier survival curve is on Figure 2. OS at 4 years was $92.5 \%$. For OS nodal stage was found to be the only statistically significant factor (HR 1.36) (Table 4). 


\section{Discussion}

The results of our institutional study are confirming the benefit of one year adjuvant trastuzumab treatment in daily practice. The magnitude of benefit was in the range of randomized studies; RFS at 4 years was $80.8 \%$ and OS $92.5 \%$, respectively.

HER2 positive $B C$ is a disease with an aggressive behaviour. Before the era of antiHER2 treatment the estimated $4-5$ years OS rate was $75-87 \%$. With the introduction of one year adjuvant trastuzumab the OS of these patients has improved significantly, according to the results of large international studies by about one third. ${ }^{12-14}$ In Slovenia, trastuzumab was rapidly implemented in the daily management after the release of these data, in the second half of 2005 already. It is well known that real life population is different to selected study population. In real world patients usually have more concomitant disease, are not as compliant as study population, cardiac follow-up is not done so often; all these factors can consequently result in worse results. The aim of our study was to assess the benefit of one year adjuvant trastuzumab treatment in our real life $B C$ patients and to compare it with the results obtained in randomized clinical studies and other published population-based studies.

In Slovenia with two million inhabitants and about 1200 newly diagnosed BCs yearly at the time of the start of this retrospective observational study we had one comprehensive cancer centre, Institute of Oncology Ljubljana. ${ }^{20}$ This is important data since the adjuvant trastuzumab treatment was preceded at this institution only and not many patients were lost from registration and follow up in the database. The median age of 313 patients included in the study was 52 years. This is comparable to international studies in which 50 to $55 \%$ of patients were younger then 50 years. ${ }^{9-14}$ Our patients had larger tumours compared to patients in international studies if a B-31 part of North American study population is excluded in which node negative patients were not included. ${ }^{11}$ Seventy-six percent of patients had tumours T1 and $\mathrm{T} 2$ and only $25 \%$ of patients had node negative disease. In comparable international studies $80-90 \%$ of patients had tumours smaller then $5 \mathrm{~cm}$. Thirty-three and $30 \%$ of patients had node negative disease in HERA and BCIRG 006 study, respectively. ${ }^{9,10}$ On the contrary, in the Dutch cohort of 479 HER2 positive BC patients $55 \%$ had node positive disease..$^{21}$ The aggressiveness of this type of $\mathrm{BC}$ could be reflected by larger volume of the disease at the first presentation and higher tumour grade.
There were $70 \%$ of high grade tumours in our population and this is comparable to North American study population. ${ }^{11}$ Only $14 \%$ of tumours were of low mitotic index. Regarding the hormonal receptor status our cohort of patients did not differ to historical cohorts. ${ }^{9-11}$ It is known that about $50 \%$ of HER2 positive BCs have positive estrogen and/or progesterone receptors and this was alike in our population. ${ }^{22}$

Adjuvant chemotherapy was as in clinical stud-

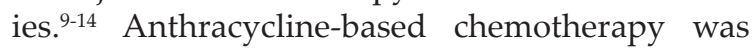
given before trastuzumab, taxane-based chemotherapy concurrently with trastuzumab. More than $90 \%$ of patients were treated with either anthracycline- or anthracycline- and taxane-based chemotherapy. Adjuvant endocrine therapy was prescribed according to international guidelines, after adjuvant chemotherapy and concomitantly with adjuvant trastuzumab. Locoregional radiotherapy was delivered to $66 \%$ of patients; the dose and the schedule were according to international guidelines. ${ }^{19}$

Tumour grade and nodal involvement were the only independent prognostic factors for the relapse (Table 3). Nodal stage was the only prognostic factors for OS. Also in North American study the tumour and nodal stage were found to be factors significantly important for both, DFS and OS. In that study especially patients with more than 10 lymph nodes involved were at the highest risk and gained the highest absolute improvement with adjuvant trastuzumab. ${ }^{11,14}$ Although RFS and DFS are not fully comparable (less events in RFS), the outcome of our non-study population is comparable to the results obtained in the international studies. ${ }^{12-14}$ The Dutch retrospective cohort study reports similar results; five years DFS and OS was $81 \%$ and $91 \%$, respectively. ${ }^{21}$

Despite indisputable efficacy of adjuvant trastuzumab treatment some questions still remain. One of them, the optimal treatment duration, was mainly resolved after obtaining results of HERA study, which showed that two years of adjuvant treatment was not more effective than one year. ${ }^{23}$ Shorter regimens like in FinHER study ${ }^{24}$ were not confirmed in PHARE and ShortHER studies. ${ }^{25,26}$ St. Gallen consensus 2013 showed almost 100\% agreement among panel discussant regarding one year lasting duration of the adjuvant trastuzumab treatment. ${ }^{19}$ There are new promising antiHER2 drugs, namely pertuzumab and trastuzumab-emtansine, which have already proven their effectiveness in the metastatic setting and will probably even improve the impressing results of trastuzumab. ${ }^{17,18}$ 
We think that our results also indicate the advantage of being treated by highly educated specialists (all treating physicians were medical oncologists), in high volume oncological center and with regular cardiac function evaluation. A prospective study of cardiotoxicity of trastuzumab in adjuvant setting is underway at our institution to show putative early and long term side effects.

\section{Conclusions}

The prognosis of HER2 positive BC has improved significantly since the introduction of antiHER2 treatment. Our results based on the treatment of real-life BC patients with one year of adjuvant trastuzumab are comparable to the results obtained in international clinical studies.

\section{References}

1. Schillani G, Era D, Cristante T, Mustacchi G, Richiardi M, Grassi L, et al. 5-HTTLPR polymorphism and anxious preoccupation in early breast cancer patients. Radiol Oncol 2012; 46: 321-7.

2. Sotiriou C, Pusztai L. Gene-expression signatures in breast cancer. N Engl J Med 2009; 360: 790-800.

3. Slamon DJ, Godolphin W, Jones LA, Holt JA, Wong SG, Keith DE, et al. Studies of the HER-2/neu proto-oncogene in human breast and ovarian cancer. Science 1989; 244: 707-12.

4. Tuzi A, Lombardi D, Crivellari D, Militello L, Perin T, La Grassa M, Massarut S, Veronesi A. Epirubicin and docetaxel as neoadjuvant treatment of hormone receptor positive, HER-2 negative breast cancer: findings from two successive phase II studies. Radiol Oncol 2013; 47: 57-62.

5. Slamon DJ, Clark GM, Wong SG, Levin WJ, Ullrich A, McGuire WL. Human breast cancer: correlation of relapse and survival with amplification of the HER-2/neu oncogene. Science 1987; 235: 177-82.

6. Slamon DJ, Leyland-Jones B, Shak S, Fuchs H, Paton V, Bajamonde A, et al. Use of chemotherapy plus a monoclonal antibody against HER2 for metastatic breast cancer that overexpresses HER2. N Engl J Med 2001; 344: 783-92.

7. Smith IE. Efficacy and safety of Herceptin in women with metastatic breast cancer: results from pivotal clinical studies. Anticancer Drugs 2001; 12(Suppl 4): S3-10.

8. Marty M, Cognetti F, Maraninchi D, Snyder R, Mauriac L, Tubiana-Hulin M, et al. Randomized phase II trial of the efficacy and safety of trastuzumab combined with docetaxel in patients with human epidermal growth factor receptor 2-positive metastatic breast cancer administered as first-line treatment: the M77001 study group. J Clin Oncol 2005; 23: 4265-74.

9. Piccart-Gebhart MJ, Procter M, Leyland-Jones B, Goldhirsch A, Untch M, Smith I, et al. Trastuzumab after adjuvant chemotherapy in HER2-positive breast cancer. N Engl J Med 2005; 353: 1659-72.

10. Slamon D, Eiermann W, Robert N. Phase III randomized trial comparing doxorubicin and cyclophosphamide followed by docetaxel (ACT) with doxorubicin and cyclophosphamide followed by docetaxel and trastuzumab (ACTH) with docetaxel, carboplatin and trastuzumab (TCH) in HER2 positive early breast cancer patients: BCIRG 006 study. [Abstract]. Breast Cancer Res Treat 2005; 94(Suppl 1): A-1.

11. Romond EH, Perez EA, Bryant J, Suman VJ, Geyer CE Jr, Davidson NE, et al. Trastuzumab plus adjuvant chemotherapy for operable HER2-positive breast cancer. N Engl J Med 2005; 353: 1673-84.
12. Gianni L, Dafni U, Gelber RD, Azambuja E, Muehlbauer S, Goldhirsch A, et al. Treatment with trastuzumab for 1 year after adjuvant chemotherapy in patients with HER2-positive early breast cancer: a 4-year follow-up of a randomised controlled trial. Lancet Oncol 2011; 12: 236-44.

13. Slamon D, Eiermann W, Robert N, Pienkowski T, Martin M, Press M, et al. Adjuvant trastuzumab in HER2-positive breast cancer. N Engl J Med 2011; 365: 1273-83.

14. Perez EA, Romond EH, Suman VJ, Jeong JH, Davidson NE, Geyer CE Jr, et al. Four-year follow-up of trastuzumab plus adjuvant chemotherapy for operable human epidermal growth factor receptor 2-positive breast cancer: joint analysis of data from NCCTG N9831 and NSABP B-31. J Clin Oncol 2011; 29: 3366-73.

15. Matos E, Čufer T. Adjuvant treatment of breast cancer patients with trastuzumab. Radiol Oncol 2007; 3: 115-22.

16. Ulhoa-Cintra A, Greenberg L, Geyer CE. The emerging role of lapatinib in HER2-positive breast cancer. Curr Oncol Rep 2008; 10: 10-7.

17. Blumenthal GM, Scher NS, Cortazar P, Chattopadhyay S, Tang S, Song P, et al First FDA approval of dual anti-HER2 regimen: pertuzumab in combination with trastuzumab and docetaxel for HER2-positive metastatic breast cancer. Clin Cancer Res 2013; 19: 1-6.

18. Ballantyne A, Dhillon S. Trastuzumab emtansine: first global approval. Drugs 2013; 73: 755-65.

19. Goldhirsch A, Winer EP, Coates AS, Gelber RD, Piccart-Gebhart M, Thürlimann B, et al. Personalizing the treatment of women with early breast cancer: highlights of the St Gallen International Expert Consensus on the Primary Therapy of Early Breast Cancer 2013. Ann Oncol 2013; 24: 2206-23.

20. Cancer in Slovenia 2009. Ljubljana: Institute of Oncology Ljubljana, Epidemiology and Cancer Registry, Cancer Registry of Republic of Slovenia; 2013.

21. Tjan-Heijnen VCG, Seferina SC, Lobbezoo DJA, Voogd AC, Dercksen MW, van den Berkmortel F, et al. Real-world use and effectiveness of adjuvant trastuzumab in 2665 consecutive breast cancer patients. [Abstract]. Cancer Res 2012; 72(24 Suppl): Nr P5-21-04.

22. Nahta R, O'Regan RM. Therapeutic implications of estrogen receptor signaling in HER2-positive breast cancers. Breast Cancer Res Treat 2012 135: $39-48$.

23. Goldhirsch A, Gelber RD, Piccart-Gebhart MJ, de Azambuja E, Procter M Suter TM, et al. 2 years versus 1 year of adjuvant trastuzumab for HER2positive breast cancer (HERA): an open-label, randomised controlled trial. Lancet 2013; 382(9897): 1021-8.

24. Joensuu H, Bono P, Kataja V, Alanko T, Kokko R, Asola R, et al. Fluorouracil, epirubicin, and cyclophosphamide with either docetaxel or vinorelbine, with or without trastuzumab, as adjuvant treatments of breast cancer: final results of the FinHer Trial. J Clin Oncol 2009; 27: 5685-92.

25. Pivot X, Romieu G, Bonnefoi H, Pierga J-Y, Kerbrat P, Guastalla J-P, et al. PHARE Trial results of subset analysis comparing 6 to 12 months of trastuzumab in adjuvant early breast cancer. [Abstract]. Cancer Res 2012; 72(24 Suppl): Nr S5-3.

26. Guarneri V, Frassoldati A, Bruzzi P, D’Amico R, Belfiglio M, Molino A, et al. Multicentric, randomized phase III trial of two different adjuvant chemotherapy regimens plus three versus twelve months of trastuzumab in patients with HER2- positive breast cancer (Short-HER Trial; NCT00629278). Clin Breast Cancer 2008; 8: 453-6. 